\title{
Book Review Electrospun Biomaterials and Related Technologies
}

\author{
Adhi Anindyajati \\ Universitas Gadjah Mada \\ Email: adhi.anindyajati@gmail.com
}

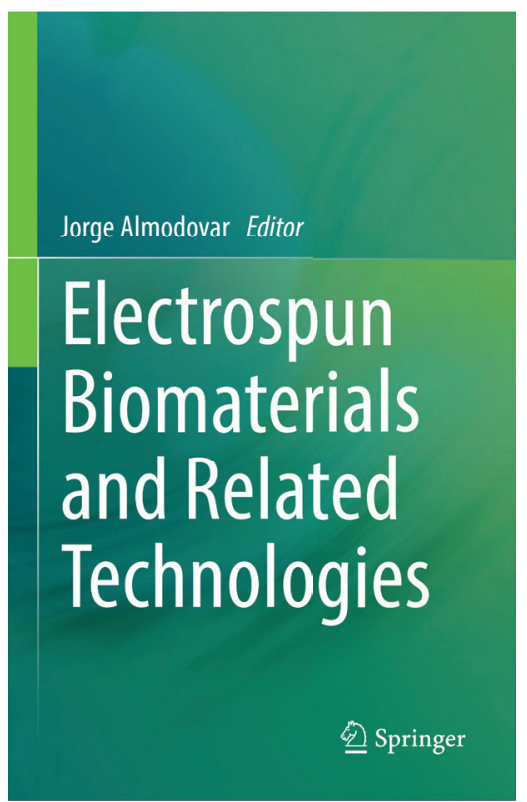
Editor : Jorge Almodovar
Penerbit : Springer International Publishing AG, Cham, Switzerland

Tahun Terbit: 2017

Tebal Buku : 282 Halaman

Electrospun Biomaterials and Related Technologies is a multi-contributed book containing review articles from worldwide authors with industry and academic background. This book is aimed to deliver a compiled overview in biomaterials electrospinning, including strategies, relevant technologies, and state-of-the-art research. The editor, Jorge Almodovar, has chemical engineering background with extensive research experiences and focus on engineering of biomimetic materials.

The book consists of nine chapters in 282 content pages. Arranged in a concise format, it delivers a comprehensive but not exhaustive reading text. The chapters cover broad range of topics in electrospinning field, including process repro-

ducibility and robustness, fibrous collagen scaffold, cellulose-based biomaterials, biopolymer nanofibres, green electrospinning, electrospun materials for cancer research, nanofibrous nerve conduits, scaffold for retinal tissue engineering, and smart material. These topics are mainly related to biomedical applications, but studies on environmental engineering are also conferred.

This first chapter discusses robustness and reproducibility of electrospinning process in order to translate lab-scale research and development into manufacturing phases. Process robustness is argued as essential to bridge gap between research achievement and market success. Several important stages for its implementation are discussed, including product design, process scaling, equipment selection and automation, process control, and quality management. The next chapter discusses the fabrication of electrospun collagen fibres for biomedical application, along with the strategies to modulate its physical and mechanical properties. It also discusses trend toward the use of nontoxic solvent, as well as applications of electrospun collagen as biomaterial for nerve, bone, dermal, tendon, ligament, and vascular graft. The third chapter describes the potential use of cellulose and its derivatives as new class of biomaterial for biomedical application. Properties and derivative of cellulose are introduced comprehensively, along with the suitable solvents for cellulose electrospinning. Examples of its biomedical applications are also described, including those for drug 
delivery, gene therapy, biosensors, wound dressing, and tissue engineering. The following chapter provides an overview on electrospinning process using biopolymers such as chitosan, cellulose, collagen, alginate, and gelatin, as well as stabilization methods for the resultant nanofibres and functionalized strategies to achieve desired characteristics. Applications of these materials are also discussed, covering both biotechnology and environmental protection field. Green electrospinning is discussed in the fifth chapter. This concept, considered as an important issue in biomaterial fabrication, is defined in this chapter, together with the influential parameters in electrospinning. Solvent selection is given a particular focus. Studies on electrospinning of polymer and composite for tissue engineering application, including those using benign solvents, are also neatly summarized.

Electrospun biomaterials are recently investigated for applications in cancer research and this topic is covered in the sixth chapter. Many roles of electrospun fibres in this area are described, including for anticancer drug and delivery, disease models for cancer, biosensors for diagnostics, and targeted stem cell delivery. The next chapter discusses the application of electrospun nanofibres to support regeneration of nervous system. Anatomies of nervous system are first explained, followed by requirements of ideal scaffold with emphasize on aligned nanofibres structure. Various fabrication methods and functionalization strategies are also discoursed. The use of electrospun scaffold for retinal treatment is also given particular attention in a chapter. Potential benefits and challenges are described, besides discussion on suitable cells for cells-based therapy, polymers used as electrospun scaffolds, cells delivery, and the delivery of drug and growth factor. The last chapter provides insight on electrospun smart materials for tissue engineering. Scaffold from this class of material is considered as more advantageous than conventional scaffolds. The concept of smart materials is explained, followed by overview on the de- velopment of piezoelectric, conductive and magnetoelectric electrospun polymers for tissue engineering.

This book conveys several interesting topics, for example robust electrospinning, green electrospinning, and smart materials. Process robustness is important consideration to transform a lab-scale research into manufacture. Electrospinning has been known to deliver promising results for biomedical application, but commercial success for medical devices based on electrospinning process appears limited. Strategies to achieve process robustness, as contained in this book, can stimulate awareness about this issue and encourage further research in electrospinning to address this concern. Green electrospinning, being a new concept, could also spark readers' interest. It is also an important topic related to safety. Electrospinning process often deals with toxic solvents, where it can be risky considering the usage of its product for biomedical application. Solvent listing and classification explained in this book can be helpful to choose less toxic solvent or for preparing necessary precautions and treatments when the use of harmful solvent is inevitable. Number of research reporting the use of less toxic solvent is still low, but those studies demonstrated promising results. It could encourage further research in electrospinning that employs nontoxic solvents. Discussion on smart materials is also thought-provoking, since capability of this class of materials to sense a stimulus and deliver predetermined response can be beneficial for developing biomaterials and medical device. Understanding on this topic opens opportunities to broaden the application of electrospinning and contribute in the advancement of electrospun biomaterials.

Papers from current research are also cited in this book, providing readers an up to date insight on research progress in electrospinning. Up to 30 papers cited in this book are published in 2017. These most current articles covers broad range of topics, including electrospinning of biopolymers such as cellulose, alginate, and chitosan, green electro- 
spinning of electrospun composite scaffolds, and electrospun materials for cancer research and retinal treatment, among others.

This book, however, can still be improved. In the first chapter, as it discusses a concept related to manufacturing system, additional discourses on optimization procedures and flexible manufacturing system (FMS) could provide valuable insights and knowledge. Optimization procedure could be important due to multivariate nature of electrospinning process that often requires the finding of optimum parameter setting. Meanwhile, FMS strategy could be useful in dealing with multiple unique or customized products. There is also redundancy in discussing basic electrospinning, in terms of electrospinning parameters and their effects, as shown in the first, third, and sixth chapter. Figure 4 in the third chapter and figure 2 in the sixth chapter also convey similar information, although they are from differ- ent sources. Furthermore, this book should be better organized by adding chapter and subchapter numberings, as well as more organized numbering for tables and figures. It would help readers in navigating their reading and referring particular topic.

Overall, this book provides the readers a broad, comprehensive, and updated knowledge in the field of biomaterial electrospinning, mainly for uses in biomedical area and also for environmental applications. Conceptual knowledge and cited studies presented in this book can provide a valuable insight to encourage further research in biomaterials for tissue engineering and regenerative medicine, particularly using electrospinning technologies.

\section{Book Bibliography}

Almodovar, J. 2017. Electrospun Biomaterials and Related Technologies, Switzerland: Springer International Publishing. 